\title{
Avaliação dos efeitos nutricionais e metabólicos da substituição de silagem de milho por ração extrusada de Capim-marandu (Urochloa brizantha) em ovinos
}

\author{
Débora Adriana de Paula Silva ${ }^{1}$; Afrânio Giroldo de Santana ${ }^{2}$; Carolina Moreira Araújo ${ }^{3 *}$; Karla Alves \\ Oliveira $^{4}$; Marco Tulio Santos Siqueira ${ }^{5}$; Gilberto de Lima Macedo Junior ${ }^{6}$
}

DOI: https://doi.org/10.35699/2447-6218.2020.19833

\begin{abstract}
Resumo:
Objetivou-se avaliar os efeitos da inclusão de níveis crescentes de volumoso extrusado (Urochloa brizantha cv Marandu) em substituição à silagem de milho sobre o consumo, digestibilidade, comportamento ingestivo e metabólitos sanguíneos em ovelhas. Foram utilizadas 20 ovelhas da raça Santa Inês com idade superior a três anos e peso corporal médio de 55,8 kg. Foi realizado um delineamento inteiramente casualizado com quatro tratamentos e cinco repetições, sendo os resultados submetidos ao estudo de regressão $(\mathrm{P}<0,05)$. Os tratamentos utilizados foram diferentes níveis de inclusão do volumoso extrusado comercial Foragge ${ }^{\circledR} 65 \%$ (F, Nuttrata Nutrição Animal ${ }^{\odot}$, Itumbiara-GO, Brasil) em substituição à silagem de milho (S), sendo: $20 \%$ de Foragge ${ }^{\circledR}$ e $80 \%$ de silagem de milho $(20 \% \mathrm{~F}: 80 \% \mathrm{~S}) ; 40 \%$ de Foragge ${ }^{\circledR}$ e $60 \%$ de silagem de milho (40\%F:60\%S); 60\% de Foragge ${ }^{\circledR}$ e $40 \%$ de silagem de milho $(60 \% \mathrm{~F}: 40 \% \mathrm{~S})$ e $80 \%$ de Foragge ${ }^{\circledR}$ e $20 \%$ de silagem de milho $(80 \% \mathrm{~F}: 20 \%$ S). Os animais foram mantidos em gaiolas metabólicas individuais, dispostas de comedouros, saleiro e bebedouros. Realizou-se estudo de digestibilidade, permitindo avaliar o consumo de matéria seca (CMS), digestibilidade, comportamento ingestivo e metabólitos sanguíneos. O CMS apresentou resposta linear positiva à inclusão do volumoso extrusado, assim com o consumo de água, peso das fezes e eficiência de ingestão, ruminação e mastigação, sem alterar a digestibilidade. Os níveis de ureia sanguínea apresentaram resposta quadrática à inclusão do volumoso extrusado. Conclui-se que a substituição parcial da silagem de milho pelo Foragge ${ }^{\circledR}$ em até $80 \%$ é recomendada, não observando-se distúrbios metabólicos ou alterações negativas na digestibilidade da matéria seca e elevação do consumo.
\end{abstract}

Palavras-chave: Extrusão. Ovis aries. Ruminação. Volumoso.

\section{Evaluation of the nutritional and metabolic effects of replacing corn silage with extruded feed of grass marandu (Urochloa brizantha) in sheep}

Abstract:

The objective was to evaluate the effects of the inclusion of increasing levels of extruded roughage (Urochloa brizantha) to replace corn silage on consumption, digestibility, ingestive behavior and blood metabolites in sheep. Twenty Santa Inês ewes over three years of age and an average body weight of $55.8 \mathrm{~kg}$ were used. A completely randomized

${ }^{1}$ Universidade Federal de Uberlândia. Uberlandia, MG. Brasil.

https://orcid.org/0000-0003-3052-0544

${ }^{2}$ Universidade Federal de Uberlândia. Uberlandia, MG. Brasil. https://orcid.org/0000-0002-3868-4474

${ }^{3}$ Universidade Federal de Uberlândia. Uberlandia, MG. Brasil. http://orcid.org/0000-0003-4648-4971

${ }^{4}$ Universidade Federal de Uberlândia. Uberlandia, MG. Brasil. http://orcid.org/0000-0002-7792-2615

${ }^{5}$ Universidade Federal de Uberlândia. Uberlandia, MG. Brasil. http://orcid.org/0000-0002-2098-8568

${ }^{6}$ Universidade Federal de Uberlândia. Uberlandia, MG. Brasil.

http://orcid.org/0000-0001-5781-7917

*Autor para correspondência: carolina.am@hotmail.com

Recebido para publicação em 27 de março de 2020. Aceito para publicação 25 de julho de 2020

e-ISSN: 2447-6218 / ISSN: 2447-6218. Atribuição CC BY. 
Silva, D. A. P., et al.

design was carried out with four treatments and five repetitions, and the results were submitted to a regression study at 95\% probability. The treatments used were different levels of inclusion of the extruded roughage Foragge ${ }^{\circledR} 65 \%$ (F) replacing corn silage (S), being: 20\% Foragge ${ }^{\circledR}$ and $80 \%$ corn silage (20\% F: $80 \%$ S ); 40\% Foragge ${ }^{\circledR}$ and $60 \%$ corn silage (40\% F: 60\% S); 60\% Foragge ${ }^{\circledR}$ and 40\% corn silage (60\% F: 40\% S) and 80\% Foragge ${ }^{\circledR}$ and $20 \%$ corn silage ( $80 \%$ F: $20 \%$ S). The animals were kept in individual metabolic cages, equipped with feeders, salt shakers and drinking fountains. A digestibility study was carried out, allowing the evaluation of dry matter intake (DMI), digestibility, ingestive behavior and blood metabolites. The DMI showed a positive linear response to the inclusion of extruded roughage, as well as water consumption, fecal weight and efficiency of ingestion, rumination and chewing, without altering digestibility. Blood urea levels showed a quadratic response to the inclusion of extruded roughage. It is concluded that the partial replacement of corn silage by Foragge ${ }^{\circledR}$ in $40 \%$, occurred in the 40F: $60 \mathrm{~S}$ treatment, is very positive, gained in efficiency, work and productivity.

Keywords: Extrusion. Ovis aries. Roughage. Rumination.

\section{Introdução}

Brasil, a principal fonte de alimento volumoso utilizado na criação de ruminantes é a forragem, uma vez que grande parte deste rebanho é produzido em sistemas à pasto. Entretanto, sabe-se que o clima predominante neste país é o tropical, sendo caracterizado por duas épocas bem distintas: água e seca. A produção de forragem é drasticamente reduzida durante a seca. Devido a isto, grande parte dos produtores fazem uso dos alimentos conservados, sendo o mais difundido a silagem de milho (Dos Santos et al., 2017).

A silagem de milho é obtida através do processo de fermentação anaeróbica dos açucares contidos na planta, realizado por bactérias. Este processo preserva a porcentagem de matéria seca da planta, assim como energia e demais nutrientes (Der Berdrosian et al., 2012). Entretanto, para a obtenção de um alimento de qualidade todos os processos envolvidos na ensilagem devem ser seguidos rigorosamente, como, plantio, época do corte, tamanho de partícula, compactação e vedação. Apesar de poder ser produzida em qualquer propriedade, é um processo que demanda planejamento, alto investimento e mão de obra.

Visando facilitar o manejo e reduzir os riscos de oscilação na qualidade dos alimentos volumosos fornecidos aos animais novos métodos de conservação de alimentos vem ganhando espaço no mercado, como é o caso dos volumosos extrusados (Oliveira et al., 2018). Segundo Guerreiro (2007) a extrusão consiste em um processo de tratamento térmico dos alimentos que com uma combinação de calor, umidade e trabalho mecânico é capaz de alterar a matéria prima dando-lhes novos formatos e novas características. O cozimento da matéria prima melhora a digestibilidade do produto, devido à desnaturação das proteínas e gelatinização do amido. Além disso, a ruptura das ligações entre carboidratos e proteínas associados à fibra a torna mais solúvel (Ramachandra e Thejaswini, 2015). Além da melhora na digestibilidade do volumoso, os alimentos extrusados podem ser facilmente armazenados e transportados nas

propriedades, facilitando o manejo e reduzindo a mão de obra.

Diante do exposto objetivou-se avaliar diferentes níveis de capim Marandu (Urochloa brizantha) extrusada em substituição à silagem milho sobre o consumo, digestibilidade, comportamento ingestivo e metabólitos sanguíneos de ovelhas adultas da raça Santa Inês.

\section{Material e métodos}

O experimento foi realizado na Fazenda Experimental Capim Branco, no setor de caprinos e ovinos, da Universidade Federal de Uberlândia, durante o mês de janeiro de 2017 com duração de 21 dias, sendo os quinze primeiros dias do experimento destinados à adaptação dos animais a ração e às gaiolas, e nos últimos seis dias foram realizadas as coletas de fezes e urina e das sobras de alimento e água. O experimento contou com a aprovação da Comissão de Ética e Utilização dos Animais (CEUA) sob o número de protocolo 092/16.

Foram utilizadas vinte ovelhas da raça Santa Inês, não gestantes, com peso corporal médio de 55,8 kg e idade superior a três anos. Todos os animais foram pesados e vermifugados com Levamisol (via oral) no primeiro dia do experimento e feita a observação da mucosa ocular, e posteriormente foram alocados em gaiolas metabólicas equipadas com comedouro, bebedouro, saleiro, piso ripado e artefato de separação de fezes e urina, localizadas em galpão de alvenaria.

Os tratamentos consistiram de diferentes níveis de inclusão de volumoso comercial extrusado, Forrage ${ }^{\circledR}$ 65\% (F) (Nuttrata Nutrição Animal ${ }^{\odot}$, Itumbiara-GO, Brasil) em substituição à silagem de milho (S). Os tratamentos foram: $20 \%$ de Forrage ${ }^{\circledR}$ e $80 \%$ de Silagem de milho (20\%F:80\%S); $40 \%$ de Forrage ${ }^{\circledR}$ e $60 \%$ de Silagem de milho (40\%F:60\%S); 60\% de Forrage ${ }^{\circledR}$ e $40 \%$ de Silagem de milho $(60 \% \mathrm{~F}: 40 \% \mathrm{~S})$ e $80 \%$ de Forrage $^{\circledR}$ e $20 \%$ de Silagem de milho (80\%F:20\%S). A alimentação foi fornecida duas vezes ao dia, às 08:00hrs e 16:00hrs, 
Avaliação dos efeitos nutricionais e metabólicos da substituição de silagem de milho por ração extrusada de Capim-marandu (Urochloa brizantha) em ovinos

e os animais tiveram livre acesso à água e sal mineral específico para ovinos.

O Forrage ${ }^{\circledR} 65 \%$ é um produto na forma extrusada, composto por $65 \%$ de capim da espécie Urochloa brizantha, cultivar Marandu, amido, farelo de girassol e premix vitamínico e mineral. Sua composição bromatológica está na Tabela 1 .

Tabela 1 - Composição bromatológica do Foragge ${ }^{\circledR} 65 \%$, silagem de milho e das dietas em função dos tratamentos.

\begin{tabular}{cccccccc}
\hline & MS & PB & FDN & FDA & NDT & Cinzas & Amido \\
\hline Foragge $^{\circledR} 65 \% *$ & 91,5 & 7,65 & 53,6 & 35,7 & 65,0 & 4,6 & 24,3 \\
Silagem de milho** & 31,2 & 8,0 & 57,11 & 36,17 & 62,52 & 10,3 & $25,63^{* * *}$ \\
\hline 20F:80S & 43,26 & 7,93 & 56,40 & 36,07 & 62,9 & - & - \\
40F:60S & 55,32 & 7,86 & 55,70 & 35,98 & 63,3 & - & - \\
60F:40S & 65,53 & 7,79 & 54,99 & 35,88 & 63,6 & - & - \\
80F:20S & 76,98 & 7,72 & 54,29 & 35,79 & 64,0 & - & - \\
\hline
\end{tabular}

MS: matéria seca; PB: proteína bruta; FDN: fibra em detergente neutro; FDA: fibra em detergente ácido; NDT: nutrientes digestíveis totais; *Valores fornecidos pelo fabricante Nutratta ${ }^{\odot}$. **Valores obtidos através de análises bromatológicas efetuadas no Laboratório de Nutrição Animal da Universidade Federal de Uberlândia. ***Dado retirado das Tabelas Brasileiras de Composição de Alimentos para Bovinos, por Valadares Filho et al., (2018).

As sobras das dietas foram mensuradas diariamente e sempre que se obteve valor igual a zero, aumentou-se a quantidade ofertada até atingir sobra equivalente a $10 \%$ do ofertado. O cálculo de CMS (consumo de matéria seca) dos alimentos foi obtido por meio da seguinte fórmula: $\mathrm{CMS}=$ (ofertado de alimento $\mathrm{x} \%$ matéria seca do ofertado) - (sobras de alimento $\mathrm{x} \%$ matéria seca das sobras).

Durante o período de coletas (cinco dias consecutivos após o período de adaptação) foram retiradas amostras de sobras e fezes de cada animal, onde ao final do período, as mesmas eram homogeneizadas e formada amostra composta para posteriores analise bromatológica e cálculo do consumo e digestibilidade da matéria seca.

Após o final do período de coleta, estas amostras eram armazenadas em freezers horizontais a $-15^{\circ} \mathrm{C}$, para conservação, em seguida foi feita a sua pré-secagem (ofertado, sobras e fezes) em estufa de circulação forçada de ar, a $55^{\circ} \mathrm{C}$ por 72 horas, até obter peso constante. Posteriormente foram trituradas, em moinho de facas tipo Willey (referência comercial), em partículas de $1 \mathrm{~mm}$. Logo após as amostras foram levadas ao laboratório onde foi feita a determinação da matéria seca das porções de ofertado, sobras e fezes, em estufa a $105^{\circ} \mathrm{C}$ por 24 horas, sendo então possível calcular a matéria seca definitiva das mesmas e teor dos nutrientes, e posteriormente, a digestibilidade aparente dos nutrientes e matéria seca através das seguintes formulas (Maynard et al., 1984):

$$
\mathrm{DA}=[\mathrm{CN}-(\text { Fez } \times \text { \%fez }) / \mathrm{CN}] \times 100
$$

Onde:

$\mathrm{CN}=$ consumo do nutriente $(\mathrm{kg})$; $\mathrm{DA}=$ digestibilidade aparente (\%); Fez = quantidade de fezes coletada $(\mathrm{kg}) ; \%$ fez $=$ teor do nutriente nas fezes $(\%)$.
O escore fecal foi avaliado todos os dias durante o período de coleta de acordo com a escala proposta por Gomes et al. (2012), no qual, na escala um (1) as fezes são ressecadas e sem brilho; na escala dois (2) as fezes são normais; na escala três (3) as fezes são ligeiramente amolecidas; na escala quatro (4) as fezes são amolecidas, perdendo o formato e coladas umas às outras (cachos de uva); na escala cinco (5) as fezes são amolecidas e sem formato normal (fezes de suínos); e na escala seis (6) as fezes são diarreicas.

O fornecimento de água foi feito todos os dias no período da manhã, em baldes plásticos, na quantidade de seis litros por animal, e também a tarde quando necessário (sempre anotando a quantidade quando fornecida), sendo as sobras de água mensuradas no dia posterior em provetas de plástico. Também se utilizou outro balde com o objetivo de mensurar a quantidade de água que evaporou, esse balde de evaporação recebia também os mesmos seis litros de água pela manhã, e na próxima manha media-se a quantidade de água que ainda restava para saber o valor do evaporado, e consequentemente, esse valor era descontado no consumo total. O cálculo do consumo de água oferecida no balde foi feito com base na diferença entre o ofertado, as sobras e o evaporado, e, o cálculo do consumo de água total foi feito somando-se a água do balde com a água contida no alimento ingerido. $\mathrm{O}$ teor de água contida no alimento foi obtido pela equação: 100 - teor de MS do alimento. Para calcular a exigência de água do animal, utilizou-se a equação proposta por Forbes (1968), que possibilita calcular quanto de água uma ovelha deve ingerir diariamente: CTA (consumo total de água) $=3,86$ x CMS - 0,99. De acordo com a fórmula, no presente estudo a quantidade de água ingerida pelos animais deve estar entre 4,25 e 6,84 litros/animal/dia.

Para a mensuração do comportamento ingestivo, que foi realizado no 5으 dia do período de coleta, os animais 
Silva, D. A. P., et al.

foram submetidos à observação visual por pessoas treinadas, em sistema de revezamento, dispostas de maneira a não incomodar os animais, por vinte e quatro horas, uma vez ao longo do período de coleta. No período noturno, o ambiente recebeu iluminação artificial, e as luzes foram mantidas acesas durante cinco dias antes da avaliação para promover a adaptação dos animais às luzes. Foram verificados, a cada cinco minutos, se os animais estavam ingerindo alimento ou água separadamente (ING), e se estavam em ruminação (RUM), ócio (ÓCIO), de acordo com a metodologia proposta por Fischer et al. (1998).

Os cálculos das atividades foram feitos em minutos por dia, admitindo que, nos cinco minutos subsequentes a cada observação, o animal permaneceu na mesma atividade. Já o tempo total gasto em mastigação (MAST) foi determinado somando-se os tempos gastos em ingestão (ING) e ruminação (RUM). As eficiência de ingestão (EIng), mastigação (EMast) e ruminação (ERum) foram obtidas segundo POLLI et al. (1996), de acordo com as equações: EIng $\left(\mathrm{g} \mathrm{min}^{-1}\right)=\mathrm{CMS} / \mathrm{Tal}$; EMast $\left(\mathrm{g} \mathrm{min}^{-1}\right)$ $=\mathrm{CMS} /$ Tmast e ERum $\left(\mathrm{g} \mathrm{min}^{-1}\right)=\mathrm{CMS} /$ Trum; em que CMS é consumo de MS ( $\mathrm{g} \mathrm{MS}$ dia $^{-1}$ ), Tal é o tempo de alimentação (min dia $^{-1}$ ), Tmast é o tempo em mastigação (min dia $^{-1}$ ) e Trum é o tempo em ruminação (min dia-1).

As coletas de sangue para avaliação dos componentes bioquímicos foram feitas no primeiro, terceiro e quinto dia (no cálculo estatístico considerou-se a média desses três dias), sempre antes do fornecimento da primeira alimentação. Para avaliação dos componentes bioquímicos foram feitas colheitas de sangue por venopunção da jugular com auxílio de tubos Vacutainer ${ }^{\circledR}$ sem anticoagulante. Os componentes bioquímicos para determinação do metabolismo energético foram: triglicerídeos e colesterol; e para determinação do metabolismo proteico foram: proteína total (PT), ureia, albumina, ácido úrico e creatinina.

Para a avaliação glicêmica a primeira coleta foi feita no 60 dia de coleta, às $8 \mathrm{~h}$ (antes da primeira refeição), $11 \mathrm{~h}, 14 \mathrm{~h}, 17 \mathrm{~h}$ e às $20 \mathrm{~h}$. No dia da avaliação glicêmica a segunda refeição somente foi ofertada após a colheita das 20h. As amostras foram coletadas por venopunção da jugular com auxílio de tubos Vacutainer ${ }^{\circledR}$ de $5 \mathrm{ml}$ contendo fluoreto e EDTA, sendo devidamente identificados para cada animal.

As amostras de sangue coletadas foram centrifugadas a 3000 rotações por minuto durante $10 \mathrm{minu-}$ tos, sendo os soros separados em alíquotas, guardados em microtubos e armazenados em freezer a $-5^{\circ} \mathrm{C}$ para posterior análise laboratorial. Todas as amostras foram processadas em analisador bioquímico automatizado Bioplus 2000 (Bioplus $^{\odot}$, Barueri-SP, Brasil), usando kit comercial da Lab Test (Labtest Diagnóstica S. A., Lagoa Santa-MG, Brasil).

Para a maioria das variáveis analisadas utilizou-se o delineamento inteiramente casualizado com quatro tratamentos e cinco repetições. Já para glicemia considerou-se delineamento inteiramente ao acaso com parcela subdivida, onde nas parcelas ficaram os tratamentos e nas sub parcelas os horários de coleta, sendo estes considerados medidas repetidas no tempo. Esta variável foi testada para a condição de esfericidade, que não foi aceita. Portanto, utilizou-se a análise de modelos mistos, em que foram avaliadas todas as estruturas de covariâncias (S) disponíveis no pacote do software SAS (SAS INSTITUTE, 2012) que modelam a dependência dos erros do modelo. Para selecionar a estrutura de covariâncias que melhor explique a correlação residual, foi utilizado o critério de informação de Akaike (AIC), sendo escolhida, para cada variável, a estrutura que resultou no menor valor de AIC após a análise (Silva et al., 2015).

Todos dados foram testados quanto a normalidade (Shapiro e Wilk, 1965) e homocedasticidade (Levene, 1960) das variâncias dos tratamentos. As variáveis normais e com variâncias homogêneas foram submetidas a análise de regressão, tendo como fator regressor o percentual do Foragge ${ }^{\circledR} 65 \%$. Nesta análise, observou-se a significância dos efeitos linear, quadrático e não significância da falta de ajuste do modelo, sendo a probabilidade de erro tipo I utilizada para tomada de decisão 5\%. Os dados referentes ao escore fecal foram analisados por estatística não paramétrica, através do teste de Kruskal e Wallis (1952) seguido pelo procedimento de Conover (1980) com nível de significância de 5\% de probabilidade de erro tipo I.

\section{Resultados e discussão}

O consumo de matéria seca (CMS) foi influenciado pela inclusão do volumoso extrusado na dieta dos animais (Tabela 2). O CMS (kg/dia) apresentou resposta linear positiva, enquanto o CMS/PC e CMS/PC ${ }^{0,75}$ apresentaram resposta quadrática em função do aumento na inclusão do Foragge ${ }^{\circledR}$ 65\%. Esse aumento nos índices de CMS, apresentado na tabela 2, é explicado por meio das características físicas e digestivas do Forrage ${ }^{\circledR} 65 \%$, ou seja, o tamanho das partículas e as características atribuídas a fibra pelo processo de extrusão.

Segundo Mertens (1992) uma das vertentes utilizadas para se explicar a regulação do consumo pelos animais são os aspectos relacionados ao alimento (nível energético, capacidade de enchimento ruminal e teor nutricional). Logo, entendemos que o processo de extrusão é responsável pelo aumento significativo no CMS. E, os fatores que podem explicar são: as partículas do volumoso extrusado são menores ( $2 \mathrm{~mm}$ em média) que as da silagem de milho e mais homogêneas, isso torna a apreensão do alimento mais fácil pelos animais e reduz o efeito de seletividade do alimento; as mudanças que ocorrem na matéria prima durante o processo de extrusão, como gelatinização do amido e desnaturação das proteínas, o que torna o alimento mais prontamente digerido no rúmen. 
Avaliação dos efeitos nutricionais e metabólicos da substituição de silagem de milho por ração extrusada de Capim-marandu (Urochloa brizantha) em ovinos

Tabela 2 - Consumo de matéria seca (CMS) em kg/dia, \% do peso corporal (PC) e do peso metabólico (PC ${ }^{0,75}$ ), e digestibilidade da matéria seca (DMS) em \%, em função da substituição da silagem de milho pelo Forrage ${ }^{\circledR} 65 \%$.

\begin{tabular}{|c|c|c|c|c|c|c|c|}
\hline & \multicolumn{4}{|c|}{ Tratamentos } & \multirow{2}{*}{$\mathbf{P}$} & \multirow{2}{*}{ MG } & \multirow{2}{*}{ CV } \\
\hline & $20 F: 80 S$ & 40F:60S & $60 \mathrm{~F}: 40 \mathrm{~S}$ & $80 \mathrm{~F}: 20 \mathrm{~S}$ & & & \\
\hline${ }^{1} \mathrm{CMS}, \mathrm{kg} / \mathrm{dia}$ & 1,36 & 2,01 & 1,79 & 2,03 & 0,0024 & 1,80 & 16,89 \\
\hline${ }^{2} \mathrm{CMS} / \mathrm{PC}, \%$ & 2,53 & 3,60 & 3,32 & 3,43 & 0,0152 & 3,22 & 16,13 \\
\hline${ }^{3} \mathrm{CMS} / \mathrm{PC}^{0,75}$ & 68,6 & 98,62 & 89,91 & 95,31 & 0,0005 & 88,11 & 15,54 \\
\hline DMS, $\%$ & 52,81 & 60,88 & 51,44 & 52,07 & 0,3698 & 54,30 & 17,21 \\
\hline
\end{tabular}

MG: média geral; CV: coeficiente de variação. ${ }^{1} \mathrm{Y}=1,359147+0,008912 \mathrm{x}, \mathrm{R}^{2}=54,97 \% ;{ }^{2} \mathrm{Y}=1,408555+0,072609 \mathrm{x}-0,000604 \mathrm{x}^{2}, \mathrm{R}^{2}=77,42 \%$; ${ }^{3} \mathrm{Y}=39,485517+1,895923 \mathrm{x}-0,015388 \mathrm{x}^{2}, \mathrm{R}^{2}=74,44 \% ; \mathrm{MG}=$ média geral; $\mathrm{CV}=$ coeficiente de variação $(\%) ; \mathrm{P}=(\mathrm{P}<0,05) ;$

De acordo com o NRC (2007) o CMS recomendado para esta categoria animal é de 1,05 kg/dia. Podemos observar que todos os tratamentos proporcionaram CMS acima do recomendado, sendo que os animais que receberam 20F:80S apresentaram um aumento de 29\% no consumo, enquanto aqueles de receberam 80F:20S apresentaram um aumento de $93 \%$. Portanto, a inclusão de volumoso extrusado na dieta proporciona aumento no CMS, quando se considera as características do alimento citadas anteriormente. Oliveira et al., (2018) trabalharam com ovelhas Santa Inês adultas, observaram CMS de 1,11 e 2,08 kg/dia para animais alimentados exclusivamente com silagem e milho e volumoso extrusado, respectivamente.

Apesar do maior consumo de matéria seca observado, a digestibilidade (DMS) dos alimentos não foi alterada, sendo a DMS média observada de $54,30 \%$, sugerindo melhor aproveitamento dos nutrientes pelos microrganismos do rumen em todos os tratamentos. Segundo Valadares Filho (2018) a digestibilidade da matéria seca média da silagem de milho produzida no Brasil encontrada na literatura é de 59,58 \%. Logo, a DMS média encontrada neste trabalho se aproxima do valor encontrado para silagem, comprovando que é possível a substituição pelo volumoso extrusado.

Segundo Oliveira (2019), o consumo de água está diretamente ligado a ingestão de matéria seca pelo animal. A tabela 3 demonstra equação linear positiva entre o consumo de água $\left(\mathrm{CH}_{2} \mathrm{O}\right)$ e a porcentagem de inclusão de Foragge ${ }^{\circledR}$ do tratamento. Isso é explicado pelo teor de matéria seca presente no do volumoso extrusado (Tabela 1), em detrimento da silagem. O volume médio de água ingerida pelos animais do experimento está dentro do recomendado, já que de acordo com Forbes (2007) o consumo de água por kg de matéria seca ingerida deve estar entre 2 e 3 litros/kg de MS ingerida.

Tabela 3 - Consumo de água em L/dia $\left(\mathrm{CH}_{2} \mathrm{O}\right)$, em $\mathrm{L} / \mathrm{kg}$ de peso corporal $\left(\mathrm{CH}_{2} \mathrm{O} / \mathrm{CMS}^{2}\right.$, volume urinário em $\mathrm{L} /$ dia (Vol. Ur), densidade urinaria (Dens. Ur.), peso de fezes na matéria natural em $\mathrm{kg} / \mathrm{dia}$ (PFMN), peso de fezes na matéria seca em $\mathrm{g} /$ dia (PFMS), matéria seca das fezes em \%, e escore fecal, \% (EF), em função da substituição da silagem de milho pelo Forrage ${ }^{\circledR} 65 \%$.

\begin{tabular}{|c|c|c|c|c|c|c|c|}
\hline & \multicolumn{4}{|c|}{ Tratamentos } & \multirow{2}{*}{$\mathbf{P}$} & \multirow{2}{*}{ MG } & \multirow{2}{*}{$\mathrm{CV}$} \\
\hline & $20 \mathrm{~F}: 80 \mathrm{~S}$ & 40F:60S & $60 \mathrm{~F}: 40 \mathrm{~S}$ & $80 \mathrm{~F}: 20 \mathrm{~S}$ & & & \\
\hline${ }^{1} \mathrm{CH}_{2} \mathrm{O}, \mathrm{L} / \mathrm{dia}$ & 1,57 & 2,47 & 3,53 & 3,6 & 0,0045 & 2,79 & 34,91 \\
\hline${ }^{2} \mathrm{CH}_{2} \mathrm{O} / \mathrm{CMS}, \mathrm{L} / \mathrm{kg}$ & 1,16 & 1,20 & 1,99 & 1,74 & 0,0085 & 1,52 & 28,44 \\
\hline VU, $L / d i a$ & 0,896 & 0,860 & 0,767 & 0,376 & 0,8759 & 0,725 & 36,85 \\
\hline${ }^{3} \mathrm{DU}, m g / d L$ & 1,0268 & 1,0258 & 1,0220 & 1,0430 & 0,0458 & 1,0294 & 1,08 \\
\hline${ }^{4} \mathrm{PFMN}, \mathrm{kg} / \mathrm{dia}$ & 1,93 & 2,33 & 2,92 & 3,12 & 0,0369 & 2,57 & 30,50 \\
\hline PFMS, $k g / d i a$ & 0,636 & 0,774 & 0,858 & 1,138 & 0,2585 & 0,852 & 32,00 \\
\hline MSF, $\%$ & 35,95 & 35,96 & 29,95 & 36,53 & 0,4785 & 34,60 & 32,53 \\
\hline$E F^{*}$ & 2,24 & 2,36 & 2,56 & 2,72 & 0,5896 & 2,47 & - \\
\hline
\end{tabular}

*Estatística não paramétrica; MG: média geral; CV: coeficiente de variação (\%); ${ }^{1} \mathrm{Y}=1,009+0,035772 \mathrm{x}, \mathrm{R}^{2}=91,53 \% ;{ }^{2} \mathrm{Y}=0,890029+0,012721 \mathrm{x}$, $\mathrm{R}^{2}=64,45 \% ;{ }^{3} \mathrm{Y}=1,045450-0,001138 \mathrm{x}+0,000014 \mathrm{x}^{2}, \mathrm{R}^{2}=85,79 \% ;{ }^{4} \mathrm{Y}=0,454752+0,007950 \mathrm{x}, \mathrm{R}^{2}=93,94 \% ; \mathrm{P}=(\mathrm{P}<0,05)$;

Segundo Hendrix (2005), a quantidade de urina excretada por pequenos ruminantes deve ficar entre 10 a $40 \mathrm{ml}$ por kg de peso vivo. Levando em conta a média do peso dos animais dos tratamentos $55,8 \mathrm{~kg}$, e o valor 
Silva, D. A. P., et al.

médio de excreção apresentado na tabela 3, chegamos em um resultado que se enquadra dentro do citado por Hendrix (2005), que é de $13 \mathrm{ml} / \mathrm{kg}$ de peso vivo.

O tratamento com maior inclusão do volumoso extrusado apresentou maior densidade urinaria, possivelmente por ter apresentado o maior CMS, conforme a tabela 2. A densidade urinaria (Dens. Ur), trata se de um indicador muito importante para a se medir a capacidade de concentração renal, sendo utilizada principalmente para analises clinicas relacionadas a doenças renais. Segundo Hendrix (2005), em pequenos ruminantes essa densidade varia de 1,020 a 1,040, sendo assim, todos os tratamentos promoveram densidade urinária dentro da normalidade. Baseando-se nos resultados acima podemos afirmar que os animais do presente estudo estavam devidamente hidratados e sem qualquer indício de problemas renais, sugerindo que os tratamentos podem substituir a silagem de milho na alimentação de ovinos.

O peso das fezes na matéria natural (PFMN) apresentou resposta linear positiva à inclusão de Foragge ${ }^{\circledR} 65 \%$ na dieta. Este resultado está diretamente relacionado ao aumento no CMS que apresentou o mesmo padrão de resposta (Tabela 2). Já o peso das fezes na matéria seca e matéria seca fecal não foram influenciados, possivelmente relacionado aos resultados obtidos para a DMS (Tabela 2). Oliveira et al., (2018) também observaram aumento no PFMN em animais adultos da raça Santa Inês alimentados com volumoso extrusado em comparação à silagem de milho.

O escore fecal (EF) é utilizado para avaliar características como, umidade, consistência e formato, sendo elas associadas a digestiblidade, com classificação de 1 a 5 (Flickinger, et al., 2003). Segundo Gomes et al., (2012) o escore fecal ideal que indica um bom funcionamento digestivo está em torno de 2 . Como a média observada no presente estudo foi de 2,47 , podemos concluir que os animais apresentavam bom funcionamento digestivo.

Com relação às variáveis analisadas para comportamento ingestivo verificou-se que apenas o tempo gasto para ruminação foi afetado pelos tratamentos apresentando equação linear negativa (Tabela 4). Essa redução no tempo (min/dia) em ruminação está relacionada com a maior inclusão do volumoso extrusado na dieta (consequentemente, menor inclusão de silagem de milho), uma vez que o mesmo é mais facilmente digerido no rúmen pelos microrganismos devido ao tamanho de partícula ser menor que a silagem de milho, demandando menor tempo para que o alimento atinja tamanho suficiente para seguir para o retículo. Oliveira et al., (2018) verificou que animais alimentados exclusivamente com volumoso extrusado ruminaram por 1,57 horas/dia, enquanto animais alimentados exclusivamente com silagem de milho ruminaram por 8,8 horas/dia.

Tabela 4 - Ingestão (ING), ruminação (RUM), ócio, mastigação (MAST), eficiência de ingestão (EIng), eficiência de ruminação (ERum), eficiência de mastigação (EMast), em função da substituição da silagem de milho pelo Forrage ${ }^{\circledR} 65 \%$.

\begin{tabular}{lcccccccc}
\hline & \multicolumn{9}{c}{ Tratamentos } & P & MG & CV \\
\cline { 2 - 5 } & $\mathbf{2 0 F}$ :80S & $\mathbf{4 0 F}$ :60S & $\mathbf{6 0 F}$ :40S & $\mathbf{8 0 F : 2 0 S}$ & & & & \\
\hline ING, min/dia & 418 & 429 & 364 & 348 & 0,1489 & 389,75 & 15,33 \\
${ }^{1}$ RUM, min/dia & 452 & 426 & 445 & 326 & 0,0256 & 412,25 & 16,93 \\
Ócio, min/dia & 570 & 585 & 685 & 766 & 0,3614 & 651,50 & 19,46 \\
MAST, min/dia & 870 & 855 & 809 & 674 & 0,3981 & 802,00 & 14,75 \\
${ }^{2}$ EIng, g/min & 3,28 & 4,78 & 4,90 & 5,98 & 0,0014 & 4,74 & 18,74 \\
${ }^{3}$ ERum, g/min & 3,10 & 4,78 & 4,02 & 6,36 & 0,0094 & 4,57 & 17,43 \\
${ }^{4}$ EMast, g/min & 1,59 & 2,38 & 2,20 & 3,06 & 0,0008 & 2,31 & 16,07 \\
\hline
\end{tabular}

MG: média geral; CV: coeficiente de variação (\%); ${ }^{1} \mathrm{Y}=502,00-1,7950 \mathrm{x}, \mathrm{R}^{2}=62,68 \% ;{ }^{2} \mathrm{Y}=2,684197+0,041171 \mathrm{x}, \mathrm{R}^{2}=91,37 \% ;{ }^{3} \mathrm{Y}=2,315840$ $+0,045098 x, R^{2}=71,74 \% ;{ }^{4} Y=1,254477+0,021123 x, R^{2}=81,20 \% ; P=(P<0,05) ;$

Segundo Van Soest (1994) animais adultos devem permanecer em torno de oito horas por dia ruminando, podendo variar entre quatro a nove horas por dia. No presente estudo, o menor tempo em ruminação foi verificado no tratamento 80F:20S e mesmo assim resultou em 5,4 horas, ou seja, dentro do valor sugerido na literatura. Neste sentido, podemos concluir que mesmo com o aumento no CMS (Tabela 2) o tempo gasto para ruminação foi reduzido, porém sem prejudicar a saúde dos animais.
Isso explica a melhora na eficiência de ingestão, ruminação e mastigação (Tabela 4), a qual também é resultado da manutenção da DMS e facilidade de apreensão do alimento por conta de seu formato. $\mathrm{O}$ aumento da taxa de passagem diminui o tempo de ruminação causando assim o aumento do consumo de matéria seca, e disponibilidade de nutrientes ao organismo do animal. Observa-se que os animais que receberam $20 \%$ de inclusão do Foragge ${ }^{\circledR}$ $65 \%$ foram capazes de ingerir $3,28 \mathrm{~g} / \mathrm{min}$ e os animais 
Avaliação dos efeitos nutricionais e metabólicos da substituição de silagem de milho por ração extrusada de Capim-marandu (Urochloa brizantha) em ovinos

que receberam $80 \%$ de Foragge ${ }^{\circledR} 65 \%$ foram capazes de ingerir $45 \%$ a mais de alimento por minuto, resultando nos CMS de 1,36 e 2,03 respectivamente (Tabela 2).

O nível de glicose sanguínea não foi alterado em função dos tratamentos e apresentou resposta linear negativa em função dos períodos avaliados (Tabela 5). A não significância para o nível de glicose nos diferentes tratamentos pode estar relacionado à semelhança existente nos níveis de NDT das dietas experimentais (Tabela
1). Segundo González (2000) em ruminantes a glicose plasmática possui como precursor o ácido graxo volátil (AGV) propionato, que após ser absorvido pelo epitélio ruminal segue para o fígado, onde é convertido em glicose através da gliconeogênese, sendo posteriormente utilizado como fonte de energia pelo animal. A produção deste AGV é aumentada em dietas com alta inclusão de concentrados, sendo assim, como as dietas do presente estudo era composta exclusivamente por volumosos, o nível de glicose sanguínea não foi alterado.

Tabela 5 - Efeito da relação volumoso extrusado: silagem de milho sobre a glicose (mg/dL).

\begin{tabular}{|c|c|c|c|c|c|c|c|c|}
\hline & \multicolumn{5}{|c|}{ Tratamentos } & \multirow{2}{*}{$\mathbf{P}$} & \multirow{2}{*}{ MG } & \multirow{2}{*}{ CV } \\
\hline & $20 F: 80 S$ & 40F:60S & & $60 \mathrm{~F}: 40 \mathrm{~S}$ & $80 F: 20 S$ & & & \\
\hline \multirow[t]{3}{*}{ Glicose, $\mathrm{mg} / d L$} & 66,28 & 64,36 & & 65,68 & 71,08 & 0,8524 & 66,85 & 14,07 \\
\hline & \multicolumn{5}{|c|}{ Horários de coleta } & \multirow{2}{*}{$\mathrm{P}$} & \multirow{2}{*}{ MG } & \multirow{2}{*}{$\mathrm{CV}$} \\
\hline & 08h & $11 \mathrm{~h}$ & $14 \mathrm{~h}$ & $17 \mathrm{~h}$ & $20 \mathrm{~h}$ & & & \\
\hline${ }^{1}$ Glicose, $m g / d L$ & 77,55 & 75,30 & 72,20 & 66,00 & 68,85 & 0,0175 & 73,69 & 12,36 \\
\hline
\end{tabular}

Valor de Referência = 29,15 - 87,18 mg/dL (Varanis, 2018); MG: média geral; CV: coeficiente de variação (\%); ${ }^{1} \mathrm{Y}=80,80-1,080 \mathrm{x}, \mathrm{R}^{2}=92,05 \%$; $\mathrm{P}=(\mathrm{P}<0,05)$;

Já a redução nos níveis de glicose plasmática ao longo do dia está relacionada ao manejo alimentar adotado, uma vez que o pico de glicose ocorreu logo após o fornecimento do alimento (8:00 horas) e reduzindo nas horas subsequentes devido ao processo de degradação e fermentação da ração no ambiente ruminal, processo este que demanda alto gasto energético, consequentemente reduzindo a concentração de glicose. Como a segunda alimentação só ocorreu após a última coleta de sangue (20:00 horas) não houve aumento nos níveis de glicose.

Dentre os metabólitos avaliados apenas ureia foi influenciada pelos tratamentos apresentando uma resposta quadrática (Tabela 6). Segundo González e Scheffer (2002), a análise do plasma sanguíneo traz uma grande segurança quanto à situação metabólica, facilitando assim a detecção de problemas metabólicos, principalmente relacionados a deficiências nutricionais.

Os níveis sanguíneos do colesterol são regulados pela adição de lipídeos na dieta (Bauchart, 1993), sendo assim, baixos níveis deste metabólito podem resultar da deficiência de alimentos energéticos, além de lesões hepato-celular ou hipertireoidismo. Enquanto o aumento de seus níveis é resultante de dietas ricas em gorduras e/ou carboidratos, hipotireoidismo e obstruções biliares (González e Silva, 2006). Os níveis deste metabólito mantiveram-se dentro do proposto na literatura para a espécie estudada, sendo assim podemos concluir que não houve excesso e nem falta de lipídeos na dieta.

Os triglicerídeos são a principal forma de armazenamento de ácidos graxos de cadeia longa, sua síntese ocorre principalmente no tecido adiposo, tendo como principal precursor o acetato. Como a dieta dos animais era exclusivamente composta por volumoso verificou-se que os níveis de triglicerídeos mantiveram próximo ao limite máximo proposto na literatura, possivelmente devido a maior produção do ácido acético.

A concentração sérica de proteínas totais está diretamente relacionada ao teor proteico advindo da dieta e pode ser um indicativo do estado nutricional do animal (Wittwer, 2000). No presente estudo os níveis de proteínas totais ficaram $26 \%$ abaixo do mínimo recomendado de acordo com valores de referência propostos por Varanis (2018). Tal fato pode estar relacionado ao baixo teor de PB das dietas experimentais, sendo esta uma característica de alimentos volumosos. Além disso, isso pode ser explicado pela alta taxa de passagem do alimento pelo rumem, devido a sua solubilidade e a sua taxa de desnaturação (Oliveira et al., 2019). Já a albumina representa $50 \%$ da proteína sérica total, mas sua alteração no organismo se dá de forma mais lenta. Falhas no funcionamento hepático, perdas durante doenças, equilíbrio hidroeletrolítico e disponibilidade de aminoácidos são problemas que podem afetar a concentração deste metabólito (González e Silva, 2006). Como no presente estudo os níveis de albumina mantiveram-se dentro do recomendado, podemos concluir que os animais se encontravam em bom estado de saúde.

A creatinina é derivada, quase em sua totalidade, do catabolismo da creatina que está contida no tecido muscular (González e Silva, 2006). Apesar da baixa atividade muscular exercida pelos animais por serem mantidos em gaiolas metabólicas a concentração deste metabólito não foi alterada. 
Silva, D. A. P., et al.

Tabela 6 - Concentração média dos metabólitos energéticos e proteicos em função dos tratamentos.

\begin{tabular}{|c|c|c|c|c|c|c|c|c|}
\hline & \multicolumn{4}{|c|}{ Tratamentos } & \multirow{2}{*}{$\mathbf{P}$} & \multirow{2}{*}{$\mathrm{VR}^{*}$} & \multirow{2}{*}{ MG } & \multirow{2}{*}{$\mathrm{CV}$} \\
\hline & 20F:80S & 40F:60S & $60 \mathrm{~F}: 40 \mathrm{~S}$ & $80 \mathrm{~F}: 20 \mathrm{~S}$ & & & & \\
\hline Triglicerídeo, $m g / d L$ & 65,26 & 53,53 & 55,53 & 59,80 & 0,5486 & $5-78$ & 58,53 & 21,37 \\
\hline Colesterol, $m g / d L$ & 45,73 & 47,46 & 43,00 & 41,53 & 0,6987 & $15-139,9$ & 44,43 & 13,16 \\
\hline $\begin{array}{l}\text { Proteínas Totais, } g / \\
d L\end{array}$ & 2,14 & 2,32 & 2,38 & 2,28 & 0,3652 & $3,10-11,4$ & 2,28 & 39,51 \\
\hline Albumina, $m g / d L$ & 3,46 & 3,53 & 2,83 & 3,37 & 0,3847 & $1,12-5,38$ & 3,30 & 18,45 \\
\hline Ácido Úrico, $m g / d L$ & 0,32 & 0,40 & 0,46 & 0,40 & 0,9963 & $0-2,9$ & 0,40 & 50,64 \\
\hline${ }^{1}$ Ureia, $m g / d L$ & 20,53 & 26,73 & 40,80 & 33,06 & 0,0489 & $12,80-100,0$ & 30,28 & 22,31 \\
\hline Creatinina, $m g / d L$ & 0,88 & 0,88 & 0,88 & 0,84 & 0,2547 & $0,40-1,80$ & 0,87 & 11,32 \\
\hline
\end{tabular}

*VR: Valor de Referência (Varanis, 2018); MG: média geral; CV: coeficiente de variação (\%); ${ }^{1} \mathrm{Y}=0,052500+1,129225 \mathrm{x}-0,008709 \mathrm{x}^{2}, \mathrm{R}^{2}=$ $80,55 \%$; $=(P<0,05)$;

O ácido úrico tem relação com o metabolismo ruminal recente, aumentando de acordo com qualidade nutricional do alimento ingerido e com a produção de proteína microbiana. Apesar dos baixos níveis de proteínas totais presente no plasma em detrimento da porcentagem de PB da dieta, o ácido úrico manteve-se dentro da normalidade. Representando assim uma boa condição de proliferação da microbiologia ruminal, permitindo concluir que o aumento da taxa de passagem promovido pela inclusão do alimento extrusado não ocasionou efeito negativo para o ambiente ruminal.

Segundo Contreras (2000) quanto maior a ingestão de proteína na ração maior será a concentração de ureia sanguínea. Sendo assim, a resposta observada para a concentração de ureia sanguínea pode estar re- lacionada ao aumento linear no CMS (Tabela 2), que consequentemente aumentou a ingestão de PB.

\section{Conclusão}

A silagem de milho pode ser substituída pelo volumoso extrusado Foragge ${ }^{\circledR} 65 \%$ em até $80 \%$, pois elevou o consumo e manteve estável a digestibilidade da matéria seca. Não se observou quaisquer distúrbios metabólicos e do comportamento ingestivo com a inclusão do Foragge ${ }^{\circledR} 65 \%$ em até $80 \%$.

\section{Aprovação do Comitê de Ética}

O experimento contou com a aprovação da Comissão de Ética e Utilização dos Animais (CEUA) sob o número de protocolo 092/16.

\section{Referências}

Bauchart, D. 1993. Lipid absorption and transport in ruminants. Journal of Dairy Science, 76: 3864-388. Doi: https://doi.org/10.3168/jds. S0022-0302(93)77728-0.

Conover, W. J. 1980. Practical Nonparametric Statistics. 2nd Edition, John Wiley \& Sons, New York.

Contreras, P. 2000. Indicadores do metabolismo proteico utilizados nos perfis metabólicos de rebanhos. P. 23-30. In: González, F. H. D.; Barcellos, J.; Patiño, H. O.; Ribeiro, L. A. eds. Perfil metabólico em ruminantes: seu uso em nutrição e doenças nutricionais. Porto Alegre: Gráfica da Universidade Federal do Rio Grande do Sul.

Dos Santos, G.; De Moraes, J. M. M.; Nussio, L. G. 2017. Custo e análise de sensibilidade na produção de silagem. Revista iPecege. 3: 39-48. Doi: 10.22167/r.ipecege.2017.1.39. Disponível em: https://www.revista. ipecege.com/Revista/article/view/100/73.

Fischer, V.; Deswysen, A. G.; Dèspres, L.; Dutilleul, P.; Lobato, J. F. P. 1998. Padrões nectemerais do comportamento ingestivo de ovinos. Revista Brasileira de Zootecnia. 27: 362-369.
Flickinger, E. A.; Schreijen, E. M. W. C.; Patil, A. R.; Hussein, H. S.; Grieshop, C. M.; Erchen, N. R.; Fahey Jr, G. C. 2003. Nutrient digestibilities, microbial populations, and protein catabolites as affected by frutan supplementation of dog diets. Journal of Animal Science, 81: 2008-2018. Doi: 10.2527/2003.8182008x. Disponível em: https:// pubmed.ncbi.nlm.nih.gov/12926783/.

Forbes, J. M. 1968. The water intake of ewes. British Journal of Nutrition, Cambridge. 22: 33-43.

Gomes, S. P.; Borges, I.; Borges, A. L. C. C.; Macedo Junior, G. L.; Campos, W. E.; Brito, T. S. 2012. Tamanho de partícula do volumoso e frequência de alimentação sobre o metabolismo energético e proteico em ovinos, considerando dietas com elevada participação de concentrado. Revista Brasileira de Saúde e Produção Animal. 13: 732-744. Disponível em: http://www.rbspa.ufba.br/index.php/rbspa/ article/viewArticle/2340.

González, F. H. D. 2000. Uso do perfil metabólico no diagnóstico de doenças metabólico nutricionais em ruminantes. In: González, F. H. D.; Barcellos, J.; Patiño, H. O.; Ribeiro, L. A. (Eds.). Perfil metabólico em ruminantes: seu uso em nutrição e doenças nutricionais. 1. ed. Porto Alegre. p. 89-106. 
Avaliação dos efeitos nutricionais e metabólicos da substituição de silagem de milho por ração extrusada de Capim-marandu (Urochloa brizantha) em ovinos

González, F. H. D.; Silva, S. C. 2006. Introdução à bioquímica clínica veterinária. Porto Alegre: Gráfica da Universidade Federal do Rio Grande do Sul. 357p.

González, F. H. D.; Sheffer, J. F. S. 2002. Perfil sanguíneo: ferramenta de análise clínica, metabólica e nutricional. In: Avaliação metabóliconutricional de vacas leiteiras por meio de fluidos corporais. Anais... 29응 Congresso de Medicina Veterinária: Gramado, Brasil.

Guerreiro, L. 2007. Produtos extrusados para consumo humano, animal e industrial. Rede de Tecnologia do Rio de Janeiro - REDETEC. 24p. Disponível em: http://www.respostatecnica.org.br/dossie-tecnico/ downloadsDT/MTcy.

Hendrix, C. M. 2005. Procedimentos laboratoriais para técnicos veterinários. 4. ed. São Paulo: Rocca. 556p.

Kruskal, W. H.; Wallis, W. A. 1952. Use of ranks in one-criterion variance analysis. Journal American Statistical Association. 47:583-621. Doi: https://doi.org/10.1080/01621459.1952.10483441.

Levene, H. 1960. Robust Test for Equality of Variances. Contributions to Probability and Statistics: Essays in Honor of Harold Hotteling', Stanford University Press, California, United States, p. 278-292.

Maynard, D. G.; Stewart, J. W. B.; Bettany, J. R. 1984. Sulfur cycling in grassland and parkland soils. Biogeochemistry. 1: 97-111. Disponível em: https://link.springer.com/article/10.1007/BF02181123.

Mertens, D. R. 1992. Análise de fibra e sua utilização na avaliação e formulação de rações. P. 188-219. In: Simpósio Internacional De Ruminantes, Anais da Reunião Anual Da Sociedade Brasileira De Zootecnia, Lavras, MG, Brasil, 29.

National Research Council - NRC. 2007. Nutrient Requeriments of Small Ruminants. 1. ed. Washington, DC, USA.

Oliveira, K. A; Macedo Junior, G. L; Silva, S. P; Araújo, C. M; Varanis, L. F. M; Sousa, L. F. 2019. Nutritional and metabolic parameters of sheep fed with extrused roughage in comparison with corn silage. Semina: Ciências Agrárias. 39:1795-1804. Doi: http://dx.doi.org/10.5433/16790359.2018v39n4p1795. Disponível em: http://www.uel.br/revistas/ uel/index.php/semagrarias/article/view/30539/23998.
Polli, V. A.; Restle, J.; Senna, D. B.; Rosa, C. E.; Aguirre, L. F.; Silva, J. H. S. 1996. Aspectos relativos à ruminação de bovinos e bubalinos em regime de confinamento. Revista Brasileira de Zootecnia, 25: 987-993. Doi: https://doi.org/10.1590/S0103-84781995000100024.

Ramachandra, H. G.; Thejaswini, M. L. 2015. Extrusion technology: a novel method of food processing. International Journal of Innovative Science, Engineering \& Technology. 2: 358-369. Disponível em: http:// ijiset.com/vol2/v2s4/IJISET_V2_I4_51.pdf.

SAS Institute Inc, JMP ${ }^{\circledR} 10$ Modeling and Multivariate Methods. 2012. Cary, NCSAS Institute Inc.

Shapiro S. S.; Wilk M. B. 1965. An Analysis of Variance Test for Normality, (Complete Samples). Biometrika. 52: 591-611. Disponível em: https://dx.doi.org/10.2307/2333709.

Silva, E. N.; Duarte, J. B.; Reis, A. J. 2015. Seleção da matriz de variânciacovariância residual na análise de ensaios varietais com medidas repetidas em cana-de-açúcar. Ciência Rural 45: 993-999. Doi: http://dx.doi. org/10.1590/0103-8478cr20141531 . Disponível em: https://www. scielo.br/scielo.php? pid $=$ S0103-84782015000600993\&script $=$ sci abstract\&tlng $=$ pt.

Valadares Filho, S. C.; Lopes, S.A.; Machado, P.A.S.; Chizzotti, M.L; Amaral, H.F.; Magalhães, K.A.; Rocha Junior, V.R.; Capelle, E.R. 2018. CQBAL 4.0. Tabelas Brasileiras de Composição de Alimentos para Bovinos. Disponível em: www.ufv.br/cqbal.

Van Soest, P. J. 1994. Nutritional ecology of the ruminant. 2.ed. Ithaca: Cornell University Press.

Varanis, L. F. M. 2018. Prospecção de metabólitos sanguíneos referenciais para ovinos em distintas categorias. Uberlândia: Universidade Federal de Uberlândia, 90f. Dissertação Mestrado. Disponível em: https:// repositorio.ufu.br/handle/123456789/21703.

Wittwer, F. 2000. Diagnóstico dos desequilíbrios metabólicos de energia em rebanhos bovinos. p. 9-22. In: González, F. H. D.; Barcellos, J. O.; Ospina, H.; Ribeiro, L. A. O. eds. Perfil metabólico em ruminantes: seu uso em nutrição e doenças nuricionais. Porto Alegre, Brasil, Gráfica da Universidade Federal do Rio Grande do Sul. Disponível em: https:// www.ufrgs.br/lacvet/restrito/pdf/perfil\%20nutricional\%20ruminantes. pdf. 